and then selectively from young DGGCs. They achieved this using the Pomc and Nestin promoters driving Cre recombinase expression. Whereas the Pomc promoter ablated essentially all 5HT1A expression, the Nestin enhancer was less effective in the younger cells, where approximately $50 \%$ still expressed the target. As expected, complete removal blocked the effects of chronic fluoxetine on a wide range of behavioral assays (noveltysuppressed feeding, elevated plus maze and forced swim test). Removal from just early DGGCs was without effect: fluoxetine retained its full repertoire of actions. In a persuasive further experiment, restoring 5-HT1A receptor expression to mature DG cells in the complete knockouts restored the effects of fluoxetine on behavior. These findings prompt new questions about the relationship between the old and young cells in this neurogenic niche.

In a parallel set of experiments using the same mouse lines, the authors showed that the same mature cells were responsible for the effects of fluoxetine on multiple aspects of neurogenesis. Rates of proliferation and the numbers and maturation rate of young adult-born DGGCs were all similarly dependent on the mature granule neuron (Fig. 1).
Having established such an important role for mature cells, the authors sought to understand how this cell type mediates these events. It has been shown that elevations in BDNF and VEGF mediate fluoxetine action on animal behavior and neurogenesis ${ }^{10,11}$. Again using the mice described above, Samuels et al. ${ }^{4}$ found that the 5HT1A receptor in the mature DGGCs elevated Bdnf and Vegf mRNA. As noted by the authors, this is quite surprising because $5 \mathrm{HT} 1 \mathrm{AR}$ activation is inhibitory and these growth factors are induced by neural activity. It is clear that we need to better understand the local effects of fluoxetine in the hippocampus.

The dorsal and ventral hippocampi have very different connections and roles. The ventral hippocampus expresses high levels of 5HT1A receptor and regulates stress responses. Fluoxetine is known to reduce the effect of stress and, again using the same mice, Samuels et al. ${ }^{4}$ showed that the $5 \mathrm{HT} 1 \mathrm{~A}$ receptor in the mature DGGCs mediates the effects of fluoxetine on corticosteroid levels. This result should prompt new work examining how fluoxetine acting through hippocampal 5HT1A receptors modulates anxiety and stress responses at the circuit level.
Samuels et al. ${ }^{4}$ bring important insight into the action of antidepressants by finding an activity in mature neurons that regulates both stress responses and neurogenesis. By learning more about the link between activity in the adult mouse brain and hippocampal tissue homeostasis, we may be able to better target specific cell types and circuits that compromise the lives of so many humans with debilitating depression.

\section{COMPETING FINANCIAL INTERESTS}

The authors declare competing financial interests: details are available at http://www.nature.com/ doifinder/10.1038/nn.4144.

1. McGrath, P.J. et al. Am. J. Psychiatry 163, 1531-1541; quiz 1666 (2006).

2. CONVERGE Consortium. Nature 523, 588-591 (2015).

3. O'Leary, O.F., Dinan, T.G. \& Cryan, J.F. Eur. J. Pharmacol. 753, 32-50 (2015).

4. Samuels, B.A. et al. Nat. Neurosci. 18, 1606-1616 (2015).

5. Malberg, J.E., Eisch, A.J., Nestler, E.J. \& Duman, R.S. J. Neurosci. 20, 9104-9110 (2000).

6. Jacobs, B.L., van Praag, H. \& Gage, F.H. Mol Psychiatry 5, 262-269 (2000).

7. Santarelli, L. et al. Science 301, 805-809 (2003).

8. Miller, B.R. \& Hen, R. Curr. Opin. Neurobiol. 30, 51-58 (2015).

9. Spalding, K.L. et al. Cell 153, 1219-1227 (2013).

10. Shirayama, Y., Chen, A.C., Nakagawa, S., Russell, D.S. \& Duman, R.S. J. Neurosci. 22, 3251-3261 (2002).

11. Warner-Schmidt, J.L. \& Duman, R.S. Proc. Natl. Acad. Sci. USA 104, 4647-4652 (2007)

\title{
Rethinking canonical cortical circuits
}

Any early graduate student can recite the rules of the canonical cortical microcircuit, which seem to be replicated throughout the brain. Input recipient layer 4 (L4) sends projections to layers $2 / 3$ (L2/3), which in turn drives layer 5 (L5). This working model exists with good reason. And yet seemingly every month (including this one) we read of departures from this standard.

On page 1631 of this issue, Pluta et al. demonstrate another non-traditional circuit in early sensory cortices of mice. Employing a remarkable combination of in vivo and in vitro extracellular and intracellular recordings along with optogenetic manipulation, the authors trace a clear and substantial pathway between L4 and L5 that bypasses superficial layers.

Optogenetic manipulation of L4 activity in vivo modulated responses in L2/3 in a way that is consistent with the canonical cortical circuit: suppression of L4 reduced L2/3 responses, whereas activation of $\mathrm{L} 4$ elevated $\mathrm{L} 2 / 3$ responses. These same manipulations concurrently resulted in the opposite change in L5, counter to what the conventional model would predict. Two major possibilities could potentially explain this sign flip: L2/3 cells might project onto local L5 inhibitory neurons or L4 might project directly to L5 inhibitory neurons.

The authors next made paired in vitro intracellular recordings to demonstrate direct

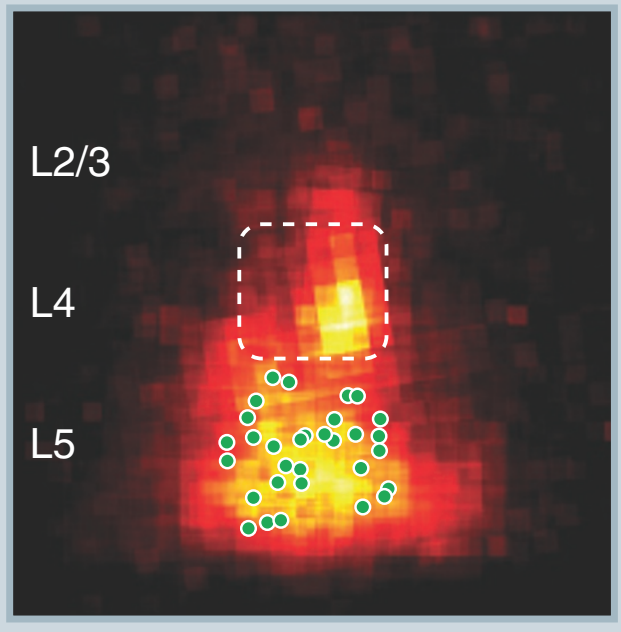
connections between L4 pyramidal cells and L5 fast-spiking cells in somatosensory cortex. The authors used focal photo-stimulation to systematically map the laminar profile of the excitatory drive onto L5 fast-spiking cells. Although every cell measured received excitatory drive from within L5, there was also a significant input from L4 (see image). In fact, a substantial fraction of cells received their strongest input from L4. There was virtually no drive from L2/3 and, in fact, disynaptic inhibition from L4 to L5 was maintained even if L2/3 was completely removed from the preparation.

Taken together, these results strongly support a direct input from L4 pyramidal cells to L5 fast-spiking cells. This suggests a revision of the model of signal flow through the cortical layers: sensory input propagates through L4 to drive L2/3 while simultaneously suppressing L5. The authors provide data suggesting that this newly identified circuit serves to sharpen tuning in the spatial domain. We might next ask whether the L4 disynaptic inhibitory connection to L5 is also present in non-sensory cortical areas, and, if so, what computation might it serve?

Neel T Dhruv 